University of Texas at El Paso

ScholarWorks@UTEP

$5-2008$

\title{
Application-Motivated Combinations of Fuzzy, Interval, and Probability Approaches, and Their Use in Geoinformatics, Bioinformatics, and Engineering
}

Vladik Kreinovich

The University of Texas at El Paso, vladik@utep.edu

Follow this and additional works at: https://scholarworks.utep.edu/cs_techrep

Part of the Computer Engineering Commons

Comments:

Technical Report: UTEP-CS-07-52a

Short version published in Proceedings of the International Conference on Information Technology InTech'07, Sydney, Australia, December 12-14, 2007, pp. 11-20. Full version published in International Journal of Automation and Control (IJAAC), 2008, Vol. 2, No. 2/3, pp. 317-339.

\section{Recommended Citation}

Kreinovich, Vladik, "Application-Motivated Combinations of Fuzzy, Interval, and Probability Approaches, and Their Use in Geoinformatics, Bioinformatics, and Engineering" (2008). Departmental Technical Reports (CS). 206.

https://scholarworks.utep.edu/cs_techrep/206

This Article is brought to you for free and open access by the Computer Science at ScholarWorks@UTEP. It has been accepted for inclusion in Departmental Technical Reports (CS) by an authorized administrator of ScholarWorks@UTEP.For more information, please contact Iweber@utep.edu. 


\title{
Application-motivated combinations of fuzzy, interval, and probability approaches, and their use in geoinformatics, bioinformatics, and engineering
}

\section{Vladik Kreinovich}

Department of Computer Science

University of Texas at El Paso

El Paso, TX 79968, USA

E-mail: vladik@utep.edu

\begin{abstract}
Most data processing techniques traditionally used in scientific and engineering practice are statistical. These techniques are based on the assumption that we know the probability distributions of measurement errors etc.

In practice, often, we do not know the distributions, we only know the bound $\Delta$ on the measurement accuracy - hence, after the get the measurement result $\widetilde{x}$, the only information that we have about the actual (unknown) value $x$ of the measured quantity is that $x$ belongs to the interval $[\widetilde{x}-\Delta, \widetilde{x}+\Delta]$. Techniques for data processing under such interval uncertainty are called interval computations; these techniques have been developed since 1950s. Many algorithms have been designed to deal with interval uncertainty.

In many practical problems, we have a combination of different types of uncertainty, where we know the probability distribution for some quantities, intervals for other quantities, and expert information for yet other quantities. It is therefore desirable to extend interval techniques to the situations when, in addition to intervals, we also have a partial probabilistic and/or expert information. We provide an overview of related algorithms, results, and remaining open problems, and we emphasize the following three application areas: computer engineering, bioinformatics, and geoinformatics.
\end{abstract}

Keywords: interval uncertainty; fuzzy uncertainty; probabilistic uncertainty; geoinformatics; bioinformatics; engineering applications.

Reference to this paper should be made as follows: Kreinovich, V. (xxxx) 'Application-motivated combinations of fuzzy, interval, and probability approaches, and their use in geoinformatics, bioinformatics, and engineering', Int. J. Automation and Control, Vol. x, No. x, pp.xxx-xxx.

Biographical notes: Vladik Kreinovich received his $\mathrm{PhD}$ in Mathematics and Physics at the Institute of Mathematics, Soviet Academy of Sciences, Novosibirsk, Russia. He is a Professor at the Department of Computer Science, University of Texas at El Paso. His research interests include interval computations, intelligent control (including fuzzy and neural approaches), and reasoning under uncertainty. He published 7 books and more than 700 research papers: book chapters, journal papers, and papers in peer-refereed international conference proceedings.

Copyright (c) 200x Inderscience Enterprises Ltd. 


\section{Main Problem}

Why indirect measurements? In many real-life situations, we are interested in the value of a physical quantity $y$ that is difficult or impossible to measure directly. Examples of such quantities are the distance to a star and the amount of oil in a given well. Since we cannot measure $y$ directly, a natural idea is to measure $y$ indirectly. Specifically, we find some easier-to-measure quantities $x_{1}, \ldots, x_{n}$ which are related to $y$ by a known relation $y=f\left(x_{1}, \ldots, x_{n}\right)$; this relation may be a simple functional transformation, or complex algorithm (e.g., for the amount of oil, numerical solution to an inverse problem). Then, to estimate $y$, we first measure the values of the quantities $x_{1}, \ldots, x_{n}$, and then we use the results $\widetilde{x}_{1}, \ldots, \widetilde{x}_{n}$ of these measurements to to compute an estimate $\widetilde{y}$ for $y$ as $\widetilde{y}=f\left(\widetilde{x}_{1}, \ldots, \widetilde{x}_{n}\right)$.

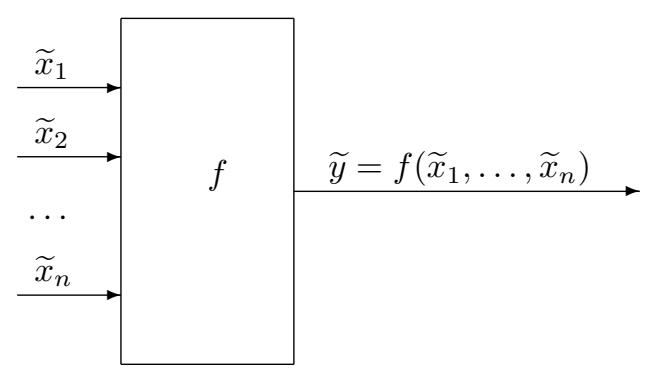

For example, to find the resistance $R$, we measure current $I$ and voltage $V$, and then use the known relation $R=V / I$ to estimate resistance as $\widetilde{R}=\widetilde{V} / \widetilde{I}$.

Computing an estimate for $y$ based on the results of direct measurements is called data processing; data processing is the main reason why computers were invented in the first place, and data processing is still one of the main uses of computers as number crunching devices.

Comment. In this paper, for simplicity, we consider the case when the relation between $x_{i}$ and $y$ is known exactly; in some practical situations, we only known an approximate relation between $x_{i}$ and $y$.

Why interval computations? From computing to probabilities to intervals. Measurements are never $100 \%$ accurate, so in reality, the actual value $x_{i}$ of $i$-th measured quantity can differ from the measurement result $\widetilde{x}_{i}$. Because of these measurement errors $\Delta x_{i} \stackrel{\text { def }}{=} \widetilde{x}_{i}-x_{i}$, the result $\widetilde{y}=f\left(\widetilde{x}_{1}, \ldots, \widetilde{x}_{n}\right)$ of data processing is, in general, different from the actual value $y=f\left(x_{1}, \ldots, x_{n}\right)$ of the desired quantity $y$.

It is desirable to describe the error $\Delta y \stackrel{\text { def }}{=} \widetilde{y}-y$ of the result of data processing. To do that, we must have some information about the errors of direct measurements.

What do we know about the errors $\Delta x_{i}$ of direct measurements? First, the manufacturer of the measuring instrument must supply us with an upper bound $\Delta_{i}$ on the measurement error. If no such upper bound is supplied, this means that no accuracy is guaranteed, and the corresponding "measuring instrument" 
is practically useless. In this case, once we performed a measurement and got a measurement result $\widetilde{x}_{i}$, we know that the actual (unknown) value $x_{i}$ of the measured quantity belongs to the interval $\mathbf{x}_{i}=\left[\underline{x}_{i}, \bar{x}_{i}\right]$, where $\underline{x}_{i}=\widetilde{x}_{i}-\Delta_{i}$ and $\bar{x}_{i}=\widetilde{x}_{i}+\Delta_{i}$.

In many practical situations, we not only know the interval $\left[-\Delta_{i}, \Delta_{i}\right]$ of possible values of the measurement error; we also know the probability of different values $\Delta x_{i}$ within this interval. This knowledge underlies the traditional engineering approach to estimating the error of indirect measurement, in which we assume that we know the probability distributions for measurement errors $\Delta x_{i}$.

In practice, we can determine the desired probabilities of different values of $\Delta x_{i}$ by comparing the results of measuring with this instrument with the results of measuring the same quantity by a standard (much more accurate) measuring instrument. Since the standard measuring instrument is much more accurate than the one use, the difference between these two measurement results is practically equal to the measurement error; thus, the empirical distribution of this difference is close to the desired probability distribution for measurement error. There are two cases, however, when this determination is not done:

- First is the case of cutting-edge measurements, e.g., measurements in fundamental science. When a Hubble telescope detects the light from a distant galaxy, there is no "standard" (much more accurate) telescope floating nearby that we can use to calibrate the Hubble: the Hubble telescope is the best we have.

- The second case is the case of measurements on the shop floor. In this case, in principle, every sensor can be thoroughly calibrated, but sensor calibration is so costly - usually costing ten times more than the sensor itself - that manufacturers rarely do it.

In both cases, we have no information about the probabilities of $\Delta x_{i}$; the only information we have is the upper bound on the measurement error.

In this case, after we performed a measurement and got a measurement result $\widetilde{x}_{i}$, the only information that we have about the actual value $x_{i}$ of the measured quantity is that it belongs to the interval $\mathbf{x}_{i}=\left[\widetilde{x}_{i}-\Delta_{i}, \widetilde{x}_{i}+\Delta_{i}\right]$. In such situations, the only information that we have about the (unknown) actual value of $y=f\left(x_{1}, \ldots, x_{n}\right)$ is that $y$ belongs to the range $\mathbf{y}=[y, \bar{y}]$ of the function $f$ over the box $\mathbf{x}_{1} \times \ldots \times \mathbf{x}_{n}$ :

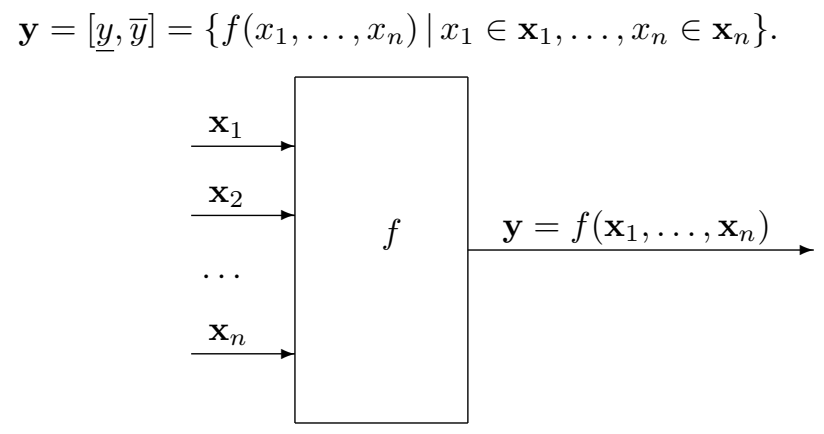

The process of computing this interval range based on the input intervals $\mathbf{x}_{i}$ is called interval computations; see, e.g., Jaulin et al. (2001); Kearfott and Kreinovich (1996). 
Interval computations techniques: brief reminder. Historically the first method for computing the enclosure for the range is the method which is sometimes called "straightforward" interval computations. This method is based on the fact that inside the computer, every algorithm consists of elementary operations (arithmetic operations, min, max, etc.). For each elementary operation $f(a, b)$, if we know the intervals $\mathbf{a}$ and $\mathbf{b}$ for $a$ and $b$, we can compute the exact range $f(\mathbf{a}, \mathbf{b})$. The corresponding formulas form the so-called interval arithmetic. For example,

$$
\begin{gathered}
{[\underline{a}, \bar{a}]+[\underline{b}, \bar{b}]=[\underline{a}+\underline{b}, \bar{a}+\bar{b}] ; \quad[\underline{a}, \bar{a}]-[\underline{b}, \bar{b}]=[\underline{a}-\bar{b}, \bar{a}-\underline{b}] ;} \\
{[\underline{a}, \bar{a}] \cdot[\underline{b}, \bar{b}]=[\min (\underline{a} \cdot \underline{b}, \underline{a} \cdot \bar{b}, \bar{a} \cdot \underline{b}, \bar{a} \cdot \bar{b}), \max (\underline{a} \cdot \underline{b}, \underline{a} \cdot \bar{b}, \bar{a} \cdot \underline{b}, \bar{a} \cdot \bar{b})] .}
\end{gathered}
$$

In straightforward interval computations, we repeat the computations forming the program $f$ step-by-step, replacing each operation with real numbers by the corresponding operation of interval arithmetic. It is known that, as a result, we get an enclosure $\mathbf{Y} \supseteq \mathbf{y}$ for the desired range.

In some cases, this enclosure is exact. In more complex cases (see examples below), the enclosure has excess width.

Example. Let us illustrate the above idea on the example of estimating the range of the function $f(x)=(x-2) \cdot(x+2)$ on the interval $x \in[1,2]$.

We start with parsing the expression for the function, i.e., describing how a computer will compute this expression; it will implement the following sequence of elementary operation:

$$
r_{1}:=x-2 ; \quad r_{2}:=x+2 ; \quad r_{3}:=r_{1} \cdot r_{2} .
$$

The main idea behind straightforward interval computations is to perform the same operations, but with intervals instead of numbers:

$$
\begin{gathered}
\mathbf{r}_{1}:=[1,2]-[2,2]=[-1,0] ; \quad \mathbf{r}_{2}:=[1,2]+[2,2]=[3,4] ; \\
\mathbf{r}_{3}:=[-1,0] \cdot[3,4]=[-4,0] .
\end{gathered}
$$

For this function, the actual range is $f(\mathbf{x})=[-3,0]$.

Comment: this is just a toy example, there are more efficient ways of computing an enclosure $\mathbf{Y} \supseteq \mathbf{y}$.

There exist more sophisticated techniques for producing a narrower enclosure, e.g., a centered form method. However, for each of these techniques, there are cases when we get an excess width. Reason: as shown in Kreinovich et al. (1997), the problem of computing the exact range is known to be NP-hard even for polynomial functions $f\left(x_{1}, \ldots, x_{n}\right)$ (actually, even for quadratic functions $f$ ).

Practical problem. In some practical situations, in addition to the lower and upper bounds on each random variable $x_{i}$, we have some additional information about $x_{i}$. So, we arrive at the following problem:

- we have a data processing algorithm $f\left(x_{1}, \ldots, x_{n}\right)$, and

- we have some information about the uncertainty with which we know $x_{i}$ (e.g., measurement errors). 
We want to know the resulting uncertainty in the result $y=f\left(x_{1}, \ldots, x_{n}\right)$ of data processing.

In interval computations, we assume that the uncertainty in $x_{i}$ can be described by the interval of possible values. In real life, in addition to the intervals, we often have some information about the probabilities of different values within this interval. What can we then do?

\section{What is the Best Way to Describe Probabilistic Uncertainty?}

How is the partial information about probabilities used in decision making? One of the main objectives of data processing is to make decisions. A standard way of making a decision is to select the action $a$ for which the expected utility (gain) is the largest possible. This is where probabilities are used: in computing, for every possible action $a$, the corresponding expected utility. To be more precise, we usually know, for each action $a$ and for each actual value of the (unknown) quantity $x$, the corresponding value of the utility $u_{a}(x)$. We must use the probability distribution for $x$ to compute the expected value $E\left[u_{a}(x)\right]$ of this utility.

In view of this application, the most useful characteristics of a probability distribution would be the ones which would enable us to compute the expected value $E\left[u_{a}(x)\right]$ of different functions $u_{a}(x)$.

Which representations are the most useful for this intended usage? General idea. Which characteristics of a probability distribution are the most useful for computing mathematical expectations of different functions $u_{a}(x)$ ? The answer to this question depends on the type of the function, i.e., on how the utility value $u$ depends on the value $x$ of the analyzed parameter.

Smooth utility functions naturally lead to moments. One natural case is when the utility function $u_{a}(x)$ is smooth. We have already mentioned, in Section I, that we usually know a (reasonably narrow) interval of possible values of $x$. So, to compute the expected value of $u_{a}(x)$, all we need to know is how the function $u_{a}(x)$ behaves on this narrow interval. Because the function is smooth, we can expand it into Taylor series. Because the interval is narrow, we can safely consider only linear and quadratic terms in this expansion and ignore higher-order terms: $u_{a}(x) \approx c_{0}+c_{1} \cdot\left(x-x_{0}\right)+c_{2} \cdot\left(x-x_{0}\right)^{2}$, where $x_{0}$ is a point inside the interval. Thus, we can approximate the expectation of this function by the expectation of the corresponding quadratic expression: $E\left[u_{a}(x)\right] \approx E\left[c_{0}+c_{1} \cdot\left(x-x_{0}\right)+c_{2} \cdot\left(x-x_{0}\right)^{2}\right]$, i.e., by the following expression: $E\left[u_{a}(x)\right] \approx c_{0}+c_{1} \cdot E\left[x-x_{0}\right]+c_{2} \cdot E\left[\left(x-x_{0}\right)^{2}\right]$. So, to compute the expectations of such utility functions, it is sufficient to know the first and second moments of the probability distribution.

In particular, if we use, as the point $x_{0}$, the average $E[x]$, the second moment turns into the variance of the original probability distribution. So, instead of the first and the second moments, we can use the mean $E$ and the variance $V$.

In decision making, non-smooth utility functions are common. In decision making, not all dependencies are smooth. There is often a threshold $x_{0}$ after which, say, a concentration of a certain chemical becomes dangerous.

This threshold sometimes comes from the detailed chemical and/or physical analysis. In this case, when we increase the value of this parameter, we see the 
drastic increase in effect and hence, the drastic change in utility value. Sometimes, this threshold simply comes from regulations. In this case, when we increase the value of this parameter past the threshold, there is no drastic increase in effects, but there is a drastic decrease of utility due to the necessity to pay fines, change technology, etc. In both cases, we have a utility function which experiences an abrupt decrease at a certain threshold value $x_{0}$.

Non-smooth utility functions naturally lead to CDFs. We want to be able to compute the expected value $E\left[u_{a}(x)\right]$ of a function $u_{a}(x)$ which changes smoothly until a certain value $x_{0}$, then drops it value and continues smoothly for $x>x_{0}$. We usually know the (reasonably narrow) interval which contains all possible values of $x$. Because the interval is narrow and the dependence before and after the threshold is smooth, the resulting change in $u_{a}(x)$ before $x_{0}$ and after $x_{0}$ is much smaller than the change at $x_{0}$. Thus, with a reasonable accuracy, we can ignore the small changes before and after $x_{0}$, and assume that the function $u_{a}(x)$ is equal to a constant $u^{+}$ for $x<x_{0}$, and to some other constant $u^{-}<u^{+}$for $x>x_{0}$.

The simplest case is when $u^{+}=1$ and $u^{-}=0$. In this case, the desired expected value $E\left[u_{a}^{(0)}(x)\right]$ coincides with the probability that $x<x_{0}$, i.e., with the corresponding value $F\left(x_{0}\right)$ of the cumulative distribution function (CDF). A generic function $u_{a}(x)$ of this type, with arbitrary values $u^{-}$and $u^{+}$, can be easily reduced to this simplest case, because, as one can easily check, $u_{a}(x)=u^{-}+\left(u^{+}-\right.$ $\left.u^{-}\right) \cdot u^{(0)}(x)$ and hence, $E\left[u_{a}(x)\right]=u^{-}+\left(u^{+}-u^{-}\right) \cdot F\left(x_{0}\right)$.

Thus, to be able to easily compute the expected values of all possible nonsmooth utility functions, it is sufficient to know the values of the CDF $F\left(x_{0}\right)$ for all possible $x_{0}$.

\section{How to Represent Partial Information about Probabilities}

General idea. In many cases, we have a complete information about the probability distributions that describe the uncertainty of each of $n$ inputs.

However, a practically interesting case is how to deal with situations when we only have partial information about the probability distributions. How can we represent this partial information?

Case of cdf. If we use cdf $F(x)$ to represent a distribution, then full information corresponds to the case when we know the exact value of $F(x)$ for every $x$. Partial information means:

- either that we only know approximate values of $F(x)$ for all $x$, i.e., that for every $x$, we only know the interval that contains $F(x)$; in this case, we get a $p$-box;

- or that we only know the values of $F(x)$ for some $x$, i.e, that we only know the values $F\left(x_{1}\right), \ldots, F\left(x_{n}\right)$ for finitely many values $x=x_{1}, \ldots, x_{n}$; in this case, we have a histogram.

It is also possible that we know only approximate values of $F(x)$ for some $x$; in this case, we have an interval-valued histogram. 
Case of moments. If we use moments to represent a distribution, then partial information means that we either know the exact values of finitely many moments, or that we know intervals of possible values of several moments.

Resulting problems. This discussion leads to a natural classification of possible problems:

- If we have complete information about the distributions of $x_{i}$, then, to get validated estimates on uncertainty of $y$, we have to use Monte-Carlo-type techniques; see, e.g., Lodwick and Jamison (2003); Lodwick, Neumaier, and Newman (2001); Moore and Lodwick (2003).

- If we have p-boxes, we can use methods from Ferson, Ginzburg, and Akcakaya (2001); Ferson et al. (2004, 2003); Kreinovich and Ferson (2006); Regan, Ferson, and Berleant (2004); Tucker and Ferson (2003).

- If we have histograms, we can use methods from Berleant et al. (2003); Berleant, Xie, and Zhang (2003); Berleant and Zhang (2004,a,b); Schroecker and Wallner (2006); Zhang and Berleant (2003).

- If we have moments, then we can use methods from Granvilliers, Kreinovich, and Mueller (2004); Kreinovich (2004); Kreinovich et al. (2004); Orshanksy et al. $(2006, a)$.

There are also additional issues, including:

- how we get these bounds for $x_{i}$ ?

- specific practical applications, like the appearance of histogram-type distributions in problems related to privacy in statistical databases.

\section{Case Study}

Practical problem. In some practical situations, in addition to the lower and upper bounds on each random variable $x_{i}$, we know the bounds $\mathbf{E}_{i}=\left[\underline{E}_{i}, \bar{E}_{i}\right]$ on its mean $E_{i}$. Indeed, in measurement practice (see, e.g., [11]), the overall measurement error $\Delta x$ is usually represented as a sum of two components:

- a systematic error component $\Delta_{s} x$ which is defined as the expected value $E[\Delta x]$, and

- a random error component $\Delta_{r} x$ which is defined as the difference between the overall measurement error and the systematic error component: $\Delta_{r} x \stackrel{\text { def }}{=}$ $\Delta x-\Delta_{s} x$.

In addition to the bound $\Delta$ on the overall measurement error, the manufacturers of the measuring instrument often provide an upper bound $\Delta_{s}$ on the systematic error component: $\left|\Delta_{s} x\right| \leq \Delta_{s}$.

This additional information is provided because, with this additional information, we not only get a bound on the accuracy of a single measurement, but we also get an idea of what accuracy we can attain if we use repeated measurements 
to increase the measurement accuracy. Indeed, the very idea that repeated measurements can improve the measurement accuracy is natural: we measure the same quantity by using the same measurement instrument several $(N)$ times, and then take, e.g., an arithmetic average $\bar{x}=\frac{\widetilde{x}^{(1)}+\ldots+\widetilde{x}^{(N)}}{N}$ of the corresponding measurement results $\widetilde{x}^{(1)}=x+\Delta x^{(1)}, \ldots, \widetilde{x}^{(N)}=x+\Delta x^{(N)}$.

- If systematic error is the only error component, then all the measurements lead to exactly the same value $\widetilde{x}^{(1)}=\ldots=\widetilde{x}^{(N)}$, and averaging does not change the value - hence does not improve the accuracy.

- On the other hand, if we know that the systematic error component is 0, i.e., $E[\Delta x]=0$ and $E[\widetilde{x}]=x$, then, as $N \rightarrow \infty$, the arithmetic average tends to the actual value $x$. In this case, by repeating the measurements sufficiently many times, we can determine the actual value of $x$ with an arbitrary given accuracy.

In general, by repeating measurements sufficiently many times, we can arbitrarily decrease the random error component and thus attain accuracy as close to $\Delta_{s}$ as we want.

When this additional information is given, then, after we performed a measurement and got a measurement result $\widetilde{x}$, then not only we get the information that the actual value $x$ of the measured quantity belongs to the interval $\mathbf{x}=[\widetilde{x}-\Delta, \widetilde{x}+\Delta]$, but we can also conclude that the expected value of $x=\widetilde{x}-\Delta x$ (which is equal to $\left.E[x]=\widetilde{x}-E[\Delta x]=\widetilde{x}-\Delta_{s} x\right)$ belongs to the interval $\mathbf{E}=\left[\widetilde{x}-\Delta_{s}, \widetilde{x}+\Delta_{s}\right]$.

If we have this information for every $x_{i}$, then, in addition to the interval $\mathbf{y}$ of possible value of $y$, we would also like to know the interval of possible values of $E[y]$. This additional interval will hopefully provide us with the information on how repeated measurements can improve the accuracy of this indirect measurement. Thus, we arrive at the following problem:

Precise formulation of the problem. Given an algorithm computing a function $f\left(x_{1}, \ldots, x_{n}\right)$ from $R^{n}$ to $R$, and values $\underline{x}_{1}, \bar{x}_{1}, \ldots, \underline{x}_{n}, \bar{x}_{n}, \underline{E}_{1}, \bar{E}_{1}, \ldots, \underline{E}_{n}, \bar{E}_{n}$, we want to find

$$
\begin{gathered}
\underline{E} \stackrel{\text { def }}{=} \min \left\{E\left[f\left(x_{1}, \ldots, x_{n}\right)\right] \mid\right. \text { all distributions of } \\
\left(x_{1}, \ldots, x_{n}\right) \text { for which } \\
x_{1} \in\left[\underline{x}_{1}, \bar{x}_{1}\right], \ldots, x_{n} \in\left[\underline{x}_{n}, \bar{x}_{n}\right] \\
\left.E\left[x_{1}\right] \in\left[\underline{E}_{1}, \bar{E}_{1}\right], \ldots E\left[x_{n}\right] \in\left[\underline{E}_{n}, \bar{E}_{n}\right]\right\} ;
\end{gathered}
$$

and $\bar{E}$ which is the maximum of $E\left[f\left(x_{1}, \ldots, x_{n}\right)\right]$ for all such distributions.

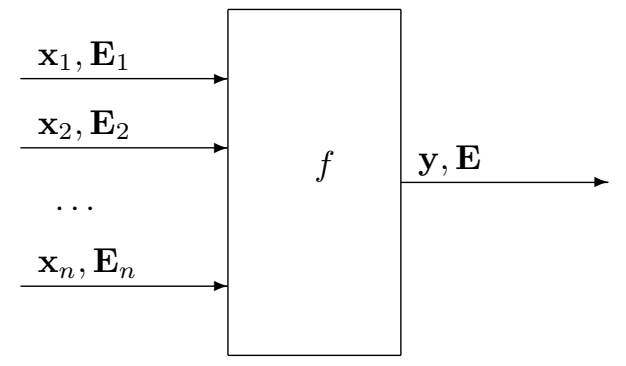


In addition to considering all possible distributions, we can also consider the case when all the variables $x_{i}$ are independent.

How we solve this problem. The main idea behind straightforward interval computations can be applied here as well. Namely, first, we find out how to solve this problem for the case when $n=2$ and $f\left(x_{1}, x_{2}\right)$ is one of the standard arithmetic operations. Then, once we have an arbitrary algorithm $f\left(x_{1}, \ldots, x_{n}\right)$, we parse it and replace each elementary operation on real numbers with the corresponding operation on quadruples $(\underline{x}, \underline{E}, \bar{E}, \bar{x})$.

To implement this idea, we must therefore know how to, solve the above problem for elementary operations.

For addition, the answer is simple. Since $E\left[x_{1}+x_{2}\right]=E\left[x_{1}\right]+E\left[x_{2}\right]$, if $y=$ $x_{1}+x_{2}$, there is only one possible value for $E=E[y]$ : the value $E=E_{1}+E_{2}$. This value does not depend on whether we have correlation or nor, and whether we have any information about the correlation. Thus, $\mathbf{E}=\mathbf{E}_{1}+\mathbf{E}_{2}$.

Similarly, the answer is simple for subtraction: if $y=x_{1}-x_{2}$, there is only one possible value for $E=E[y]$ : the value $E=E_{1}-E_{2}$. Thus, $\mathbf{E}=\mathbf{E}_{1}-\mathbf{E}_{2}$.

For multiplication, if the variables $x_{1}$ and $x_{2}$ are independent, then $E\left[x_{1} \cdot x_{2}\right]=$ $E\left[x_{1}\right] \cdot E\left[x_{2}\right]$. Hence, if $y=x_{1} \cdot x_{2}$ and $x_{1}$ and $x_{2}$ are independent, there is only one possible value for $E=E[y]$ : the value $E=E_{1} \cdot E_{2}$; hence $\mathbf{E}=\mathbf{E}_{1} \cdot \mathbf{E}_{2}$.

The first non-trivial case is the case of multiplication in the presence of possible correlation. When we know the exact values of $E_{1}$ and $E_{2}$, the solution to the above problem is as follows:

Theorem 1. For multiplication $y=x_{1} \cdot x_{2}$, when we have no information about the correlation,

$$
\begin{gathered}
\underline{E}=\max \left(p_{1}+p_{2}-1,0\right) \cdot \bar{x}_{1} \cdot \bar{x}_{2}+\min \left(p_{1}, 1-p_{2}\right) \cdot \bar{x}_{1} \cdot \underline{x}_{2}+ \\
\min \left(1-p_{1}, p_{2}\right) \cdot \underline{x}_{1} \cdot \bar{x}_{2}+\max \left(1-p_{1}-p_{2}, 0\right) \cdot \underline{x}_{1} \cdot \underline{x}_{2} ; \\
\bar{E}=\min \left(p_{1}, p_{2}\right) \cdot \bar{x}_{1} \cdot \bar{x}_{2}+\max \left(p_{1}-p_{2}, 0\right) \cdot \bar{x}_{1} \cdot \underline{x}_{2}+ \\
\max \left(p_{2}-p_{1}, 0\right) \cdot \underline{x}_{1} \cdot \bar{x}_{2}+\min \left(1-p_{1}, 1-p_{2}\right) \cdot \underline{x}_{1} \cdot \underline{x}_{2},
\end{gathered}
$$

where $p_{i} \stackrel{\text { def }}{=}\left(E_{i}-\underline{x}_{i}\right) /\left(\bar{x}_{i}-\underline{x}_{i}\right)$.

Theorem 2. For multiplication under no information about dependence, to find $\underline{E}$, it is sufficient to consider the following combinations of $p_{1}$ and $p_{2}$ :

- $p_{1}=\underline{p}_{1}$ and $p_{2}=\underline{p}_{2} ; p_{1}=\underline{p}_{1}$ and $p_{2}=\bar{p}_{2} ; p_{1}=\bar{p}_{1}$ and $p_{2}=\underline{p}_{2} ; p_{1}=\bar{p}_{1}$ and $p_{2}=\bar{p}_{2}$;

- $p_{1}=\max \left(\underline{p}_{1}, 1-\bar{p}_{2}\right)$ and $p_{2}=1-p_{1}$ (if $\left.1 \in \mathbf{p}_{1}+\mathbf{p}_{2}\right)$; and

- $p_{1}=\min \left(\bar{p}_{1}, 1-\underline{p}_{2}\right)$ and $p_{2}=1-p_{1}\left(\right.$ if $\left.1 \in \mathbf{p}_{1}+\mathbf{p}_{2}\right)$.

The smallest value of $\underline{E}$ for all these cases is the desired lower bound $\underline{E}$.

Theorem 3. For multiplication under no information about dependence, to find $\bar{E}$, it is sufficient to consider the following combinations of $p_{1}$ and $p_{2}$ :

- $p_{1}=\underline{p}_{1}$ and $p_{2}=\underline{p}_{2} ; p_{1}=\underline{p}_{1}$ and $p_{2}=\bar{p}_{2} ; p_{1}=\bar{p}_{1}$ and $p_{2}=\underline{p}_{2} ; p_{1}=\bar{p}_{1}$ and $p_{2}=\bar{p}_{2}$; 
- $p_{1}=p_{2}=\max \left(\underline{p}_{1}, \underline{p}_{2}\right)\left(\right.$ if $\left.\mathbf{p}_{1} \cap \mathbf{p}_{2} \neq \emptyset\right)$; and

- $p_{1}=p_{2}=\min \left(\bar{p}_{1}, \bar{p}_{2}\right)\left(\right.$ if $\left.\mathbf{p}_{1} \cap \mathbf{p}_{2} \neq \emptyset\right)$.

The largest value of $\bar{E}$ for all these cases is the desired upper bound $\bar{E}$.

For the inverse $y=1 / x_{1}$, the finite range is possible only when $0 \notin \mathbf{x}_{1}$. Without losing generality, we can consider the case when $0<\underline{x}_{1}$. In this case, we get the following bound:

Theorem 4. For the inverse $y=1 / x_{1}$, the range of possible values of $E$ is $\mathbf{E}=$ $\left[1 / E_{1}, p_{1} / \bar{x}_{1}+\left(1-p_{1}\right) / \underline{x}_{1}\right]$.

(Here $p_{1}$ denotes the same value as in Theorem 1).

Similar formulas can be produced for max and min, and also for the cases when there is a strong correlation between $x_{i}$ : namely, when $x_{1}$ is (non-strictly) increasing or decreasing in $x_{2}$; see, e.g., Kreinovich (2004).

Additional results. The above techniques assume that we already know the moments etc., but how can we compute them based on the measurement results? For example, when we have only interval ranges $\left[\underline{x}_{i}, \bar{x}_{i}\right]$ of sample values $x_{1}, \ldots, x_{n}$, what is the interval $[\underline{V}, \bar{V}]$ of possible values for the variance $V$ of these values?

It turns out that most such problems are computationally difficult (to be more precise, NP-hard), and we provide feasible algorithms that compute these bounds under reasonable easily verifiable conditions Ferson et al. (2005); Kreinovich et al. (2006).

Challenges. What is, in addition to intervals and first moments, we also know second moments (this problem is important for design of computer chips):

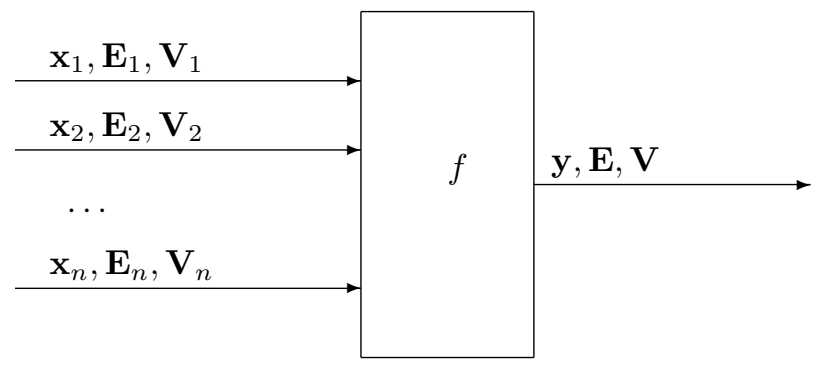

What if, in addition to moments, we also know p-boxes?

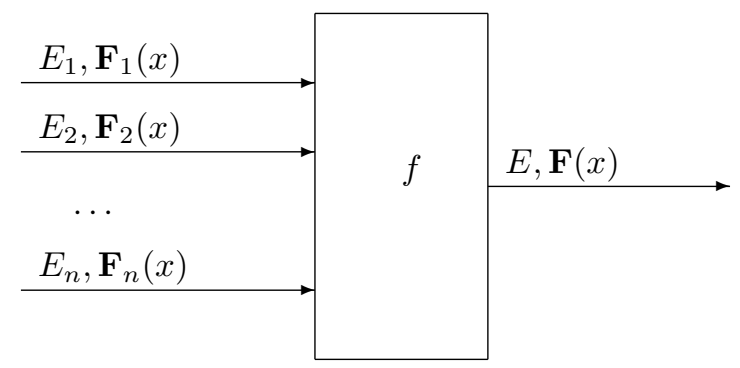




\section{Fuzzy Uncertainty: In Brief}

In the fuzzy case, for each value of measurement error $\Delta x_{i}$, we describe the degree $\mu_{i}\left(\Delta x_{i}\right)$ to which this value is possible.

For each degree of certainty $\alpha$, we can determine the set of values of $\Delta x_{i}$ that are possible with at least this degree of certainty - the $\alpha$-cut $\{x \mid \mu(x) \geq \alpha\}$ of the original fuzzy set. Vice versa, if we know $\alpha$-cuts for every $\alpha$, then, for each object $x$, we can determine the degree of possibility that $x$ belongs to the original fuzzy set Dubois and Prade (1978); Klir and Yuan (1995); Moore and Lodwick (2003); Nguyen and Kreinovich (1996); Nguyen and E. A. Walker (2006). A fuzzy set can be thus viewed as a nested family of its $\alpha$-cuts.

If instead of a (crisp) interval $\mathbf{x}_{i}$ of possible values of the measured quantity, we have a fuzzy set $\mu_{i}(x)$ of possible values, then we can view this information as a family of nested intervals $\mathbf{x}_{i}(\alpha)-\alpha$-cuts of the given fuzzy sets.

Our objective is then to compute the fuzzy number corresponding to this the desired value $y=f\left(x_{1}, \ldots, x_{n}\right)$. In this case, for each level $\alpha$, to compute the $\alpha$-cut of this fuzzy number, we can apply the interval algorithm to the $\alpha$-cuts $\mathbf{x}_{i}(\alpha)$ of the corresponding fuzzy sets. The resulting nested intervals form the desired fuzzy set for $y$.

\section{Case Study: Chip Design}

Decreasing clock cycle: a practical problem. In chip design, one of the main objectives is to decrease the chip's clock cycle. It is therefore important to estimate the clock cycle on the design stage.

The clock cycle of a chip is constrained by the maximum path delay over all the circuit paths $D \stackrel{\text { def }}{=} \max \left(D_{1}, \ldots, D_{N}\right)$, where $D_{i}$ denotes the delay along the $i$-th path. Each path delay $D_{i}$ is the sum of the delays corresponding to the gates and wires along this path. Each of these delays, in turn, depends on several factors such as the variation caused by the current design practices, environmental design characteristics (e.g., variations in temperature and in supply voltage), etc.

Traditional (interval) approach to estimating the clock cycle. Traditionally, the delay $D$ is estimated by using the worst-case analysis, in which we assume that each of the corresponding factors takes the worst possible value (i.e., the value leading to the largest possible delays). As a result, we get the time delay that corresponds to the case when all the factors are at their worst.

It is necessary to take probabilities into account. The worst-case analysis does not take into account that different factors come from independent random processes. As a result, the probability that all these factors are at their worst is extremely small. For example, there may be slight variations of delay time from gate to gate, and this can indeed lead to gate delays. The worst-case analysis considers the case when all these random variations lead to the worst case; since these variations are independent, this combination of worst cases is highly improbable.

As a result, the current estimates of the chip clock time are over-conservative, over up to $30 \%$ above the observed clock time. Because of this over-estimation, the clock time is set too high - i.e., the chips are usually over-designed and under- 
performing; see, e.g., Chinnery and Keutzer (2002). To improve the performance, it is therefore desirable to take into account the probabilistic character of the factor variations.

How the desired delay $D$ depends on the parameters. The variations in the each gate delay $d$ are caused by the difference between the actual and the nominal values of the corresponding parameters. It is therefore desirable to describe the resulting delay $d$ as a function of these differences $x_{1}, \ldots, x_{n}$. Since these differences are usually small, we can safely ignore quadratic (and higher order) terms in the Taylor expansion of the dependence of $d$ on $x_{j}$ and assume that the dependence of each delay $d$ on these differences can be described by a linear function.

As a result, each path delay $D_{i}$ - which, as we have mentioned, is the sum of delays at different gates and wires - can also be described as a linear function of these differences, i.e., as $D_{i}=a_{i}+\sum_{j=1}^{n} a_{i j} \cdot x_{j}$ for some coefficients $a_{i}$ and $a_{i j}$. Thus, the desired maximum delay $D=\max _{i} D_{i}$ has the form

$$
D=\max _{i}\left(a_{i}+\sum_{j=1}^{n} a_{i j} \cdot x_{j}\right) .
$$

How we can describe such functions in general terms. In this section, we will use two properties of the time delay. First, we will use the fact that the time delay is always non-negative; second, we will use the fact that the dependence (1) is convex.

Let us recall that a function $f: R^{m} \rightarrow R$ is called convex if

$$
f(\alpha \cdot x+(1-\alpha) \cdot y) \leq \alpha \cdot f(x)+(1-\alpha) \cdot f(y)
$$

for every $x, y \in R^{m}$ and for every $\alpha \in(0,1)$. It is known that the maximum of several linear functions is convex, so the function (1) is convex. Vice versa, every convex function can be approximated, with an arbitrary accuracy, by maxima of linear functions - i.e., by expressions of type (1).

So, in general terms, we can say that we are interested in the robust statistical properties of the value $y=F\left(x_{1}, \ldots, x_{n}\right)$, where $F$ is a non-negative convex function of the variables $x_{j}$.

Our objective. We want to find the smallest possible value $y_{0}$ such that for all possible distributions consistent with the known information, we have $y \leq y_{0}$ with the probability $\geq 1-\varepsilon$ (where $\varepsilon>0$ is a given small probability).

What information we can use. What information can we use for these estimations? We can safely assume that different factors $x_{j}$ are statistically independent. About some of the variables $x_{j}$, we know their exact statistical characteristics; about some other variables $x_{j}$, we only know their interval ranges $\left[\underline{x}_{j}, \bar{x}_{j}\right]$ and their means $E_{j}$.

Additional property: the dependency is non-degenerate. We only have partial information about the probability distribution of the variables $x_{j}$. For each possible probability distribution $p$, we can find the largest value $y_{p}$ for which, for 
this distribution, $y \leq y_{p}$ with probability $\geq 1-\varepsilon$. The desired value $y_{0}$ is the largest of the values $y_{p}$ corresponding to different probability distributions $p: y_{0}=\sup _{p \in P} y_{p}$, where $P$ denotes the class of probability distributions $p$ which are consistent with the known information.

If we learn some additional information about the distribution of $x_{j}-$ e.g., if we learn that $x_{j}$ actually belongs to a proper subinterval of the original interval $\left[\underline{x}_{j}, \bar{x}_{j}\right]$ - we thus decrease the class $P$ of distributions $p$ which are consistent with this information, to a new class $P^{\prime} \subset P$. Since the class has decreased, the new value $y_{0}^{\prime}=\sup _{p \in P^{\prime}} y_{p}$ is the maximum over a smaller set and thus, cannot be larger than the original value $y_{0}: y_{0}^{\prime} \leq y_{0}$.

From the purely mathematical viewpoint, it is, in principle, possible that the desired value $y$ does not actually depend on some of the variables $x_{j}$. In this case, if we narrow down the interval of possible values of the corresponding variable $x_{j}$, this will not change the resulting value $y_{0}$.

For the chip design problem, it is reasonable to assume that such variables have already been weeded out, and that the resulting function $F\left(x_{1}, \ldots, x_{n}\right)$ is non-degenerate in the sense that every time we narrow down one of the intervals $\left[\underline{x}_{j}, \bar{x}_{j}\right]$, the resulting value $y_{0}$ actually decreases: $y_{0}^{\prime}<y_{0}$.

As a result, we arrive at the following problem.

\section{Formulation of the problem and the main result.}

\section{GIVEN:}

- natural numbers $n$, and $k \leq n$;

- a real number $\varepsilon>0$;

- a function $y=F\left(x_{1}, \ldots, x_{n}\right)$ (algorithmically defined) such that for every combination of values $x_{k+1}, \ldots, x_{n}$, the dependence of $y$ on $x_{1}, \ldots, x_{k}$ is convex;

- $n-k$ probability distributions $x_{k+1}, \ldots, x_{n}-$ e.g., given in the form of cumulative distribution function (cdf) $F_{j}(x), k+1 \leq j \leq n$;

- $k$ intervals $\mathbf{x}_{1}, \ldots, \mathbf{x}_{k}$, and

- $k$ values $E_{1}, \ldots, E_{k}$,

such that for every $x_{1} \in\left[\underline{x}_{1}, \bar{x}_{1}\right], \ldots, x_{k} \in\left[\underline{x}_{k}, \bar{x}_{k}\right]$, we have $F\left(x_{1}, \ldots, x_{n}\right) \geq 0$ with probability 1 .

TAKE: all possible joint probability distributions on $R^{n}$ for which:

- all $n$ random variables are independent;

- for each $j$ from 1 to $k, x_{j} \in \mathbf{x}_{j}$ with probability 1 and the mean value of $x_{j}$ is equal to $E_{j}$;

- for $j>k$, the variable $x_{j}$ has a given distribution $F_{j}(x)$. 
FIND: the smallest possible value $y_{0}$ such that for all possible distributions consistent with the known information, we have $y \stackrel{\text { def }}{=} F\left(x_{1}, \ldots, x_{n}\right) \leq y_{0}$ with probability $\geq 1-\varepsilon$.

PROVIDED: that the problem is non-degenerate in the sense that if we narrow down one of the intervals $\mathbf{x}_{j}$, the value $y_{0}$ decreases.

The following result explains how we can compute this value $y_{0}$.

Theorem 5. Orshanksy et al. (2006) The desired value $y_{0}$ is attained when for each $j$ from 1 to $k$, we use a 2-point distribution for $x_{j}$, in which:

- $x_{j}=\underline{x}_{j}$ with probability $\underline{p}_{j} \stackrel{\text { def }}{=} \frac{\bar{x}_{j}-E_{j}}{\bar{x}_{j}-\underline{x}_{j}}$.

- $x_{j}=\bar{x}_{j}$ with probability $\bar{p}_{j} \stackrel{\text { def }}{=} \frac{E_{j}-\underline{x}_{j}}{\bar{x}_{j}-\underline{x}_{j}}$.

Comment. The proof of Theorem 5 is given in the special (last) subsection of this section.

Resulting algorithm for computing $y_{0}$. Because of Theorem 5, we can compute the desired value $y_{0}$ by using the following Monte-Carlo simulation:

- We set each value $x_{j}, 1 \leq j \leq k$, to be equal to $\bar{x}_{j}$ with probability $\bar{p}_{j}$ and to the value $\underline{x}_{j}$ with the probability $\underline{p}_{j}$.

- We simulate the values $x_{j}, k<j \leq n$, as random variables distributed according to the distributions $F_{j}(x)$.

- For each simulation $s, 1 \leq s \leq N_{i}$, we get the simulated values $x_{j}^{(s)}$, and then, a value $y^{(s)}=F\left(x_{1}^{(s)}, \ldots, x_{n}^{(s)}\right)$. We then sort the resulting $N_{i}$ values $y^{(s)}$ into an increasing sequence

$$
y_{(1)} \leq y_{(2)} \leq \ldots \leq y_{\left(N_{i}\right)}
$$

and take, as $y_{0}$, the $N_{i} \cdot(1-\varepsilon)$-th term $y_{\left(N_{i} \cdot(1-\varepsilon)\right)}$ in this sorted sequence.

Comment about Monte-Carlo techniques. Before presenting the algorithm for computing the upper bound on $y_{0}$, let us remark that some readers may feel uncomfortable with the use of Monte-Carlo techniques. This discomfort comes from the fact that in the traditional statistical approach, when we know the exact probability distributions of all the variables, Monte-Carlo methods - that simply simulate the corresponding distributions - are inferior to analytical methods. This inferiority is due to two reasons:

- First, by design, Monte-Carlo methods are approximate, while analytical methods are usually exact.

- Second, the accuracy provided by a Monte-Carlo method is, in general, proportional to $\sim 1 / \sqrt{N_{i}}$, where $N_{i}$ is the total number of simulations. Thus, to achieve reasonable quality, we often need to make a lot of simulations - as a result, the computation time required for a Monte-Carlo method becomes much longer than for an analytical method. 
In robust statistic, there is often an additional reason to be uncomfortable about using Monte-Carlo methods:

- Practitioners use these methods by selecting a finite set of distributions from the infinite class of all possible distributions, and running simulations for the selected distributions.

- Since we do not test all the distributions, this practical heuristic approach sometimes misses the distributions on which the minimum or maximum of the corresponding distribution is actually attained.

In our case, we also select a finite collection of distributions from the infinite set. However, in contrast to the heuristic (un-justified) selection - which is prone to the above criticism, our selection is justified. Theorem 5 guarantees that the values corresponding to the selected distributions indeed provide the desired value $y_{0}-$ the largest over all possible distributions $p \in P$.

In such situations, where a justified selection of Monte-Carlo methods is used to solve a problem of robust statistics, such Monte-Carlo methods often lead to faster computations than known analytical techniques. The speed-up caused by using such Monte-Carlo techniques is one of the main reasons why they were invented in the first place - to provide fast estimates of the values of multi-dimensional integrals. Many examples of efficiency of these techniques are given, e.g., in Rajasekaran et al. (2001); in particular, examples related to estimating how the uncertainty of inputs leads to uncertainty of the results of data processing are given in Trejo and Kreinovich (2001).

Proof of Theorem 5. By definition, $y_{0}$ is the largest value of $y_{p}$ over all possible distributions $p \in P$. This means that for the given $y_{0}$, for all possible distributions $p \in P$, we have $\operatorname{Prob}\left(D \leq y_{0}\right) \geq 1-\varepsilon$. Let $p \in P$ be the "worst-case" distribution, i.e., the distribution for which the probability $\operatorname{Prob}\left(D \leq y_{0}\right)$ is the smallest. Let us show that this "worst case" occurs when all $k$ variables $x_{1}, \ldots, x_{k}$ have the 2-point distributions described in Theorem 5 .

Let us fix the value $j \leq k$ and show that in the "worst case", $x_{j}$ indeed has the desired 2-point distribution. Without losing generality, we can take $j=1$. Let us fix the distributions for $x_{2}, \ldots, x_{k}$ as in the worst case. Then, the fact that the probability $\operatorname{Prob}\left(D \leq y_{0}\right)$ is the smallest means that if we replace the worst-case distribution for $x_{1}$ with some other distribution, we can only increase this probability. In other words, when we correspondingly fix the distributions for $x_{2}, \ldots, x_{k}$, the probability $\operatorname{Prob}\left(D \leq y_{0}\right)$ attains the smallest possible value at the desired distribution for $x_{1}$.

In reality, the distribution for $x_{1}$ is located on an interval $\mathbf{x}_{1}=\left[\underline{x}_{1}, \bar{x}_{1}\right]$, i.e., on a set with infinitely many points. However, with an arbitrary large value $N$ (and thus, for an arbitrarily small discretization error $\left.\delta=\left(\bar{x}_{1}-\underline{x}_{1}\right) / N\right)$, we can assume that all the distributions are located on a finite grid of values

$$
v_{0} \stackrel{\text { def }}{=} \underline{x}_{1}, \quad v_{1} \stackrel{\text { def }}{=} \underline{x}_{1}+\delta, \quad v_{2} \stackrel{\text { def }}{=} \underline{x}_{1}+2 \delta, \ldots, v_{N}=\bar{x}_{1} .
$$

The smaller $\delta$, the better this approximation. Thus, without losing generality, we can assume that the distribution of $x_{1}$ is located on finitely many points $v_{i}$.

In this approximation, the probability distribution for $x_{1}$ can be described by the probabilities $q_{i} \stackrel{\text { def }}{=} p_{1}\left(v_{i}\right)$ of different values $v_{i}$. 
The minimized probability $\operatorname{Prob}\left(D \leq y_{0}\right)$ can be described as the sum of the probabilities of different combinations $\left(x_{1}, \ldots, x_{n}\right)$ over all the combinations for which $D\left(x_{1}, \ldots, x_{n}\right) \leq y_{0}$. We assumed that all the variables $x_{j}$ are independent. Thus, the probability of each combination $\left(x_{1}, \ldots, x_{n}\right)$ is equal to the product of the corresponding probabilities $p_{1}\left(x_{1}\right) \cdot p_{2}\left(x_{2}\right) \cdot \ldots$ Since the probability distributions for $x_{2}, \ldots$ are fixed, the minimized probability is thus a linear combination of probabilities $p_{1}\left(v_{i}\right)$, i.e., of the probabilities $q_{i}$. In other words, the minimized probability has the form $\sum_{i=0}^{N} c_{i} \cdot q_{i}$ for some coefficients $c_{i}$.

By describing the probability distribution on $x_{1}$ via the probabilities $q_{i}=p_{1}\left(v_{i}\right)$ of different values $v_{i} \in\left[\underline{x}_{1}, \bar{x}_{1}\right]$, we automatically restrict ourselves to distributions which are located on this interval. The only restrictions that we have on the probability distribution of $x_{1}$ is that it is a probability distribution, i.e., that $q_{i} \geq 0$ for all $i$ and $\sum_{i=0}^{N} q_{i}=1$, and that the mean value of this distribution is equal to $E_{1}$, i.e., that $\sum_{i=0}^{N} q_{i} \cdot v_{i}=E_{1}$. Thus, the worst-case distribution for $x_{1}$ is a solution to the following linear programming problem:

$$
\text { Minimize } \sum_{i=0}^{N} c_{i} \cdot q_{i}
$$

under the constraints

$$
\sum_{i=0}^{N} q_{i}=1, \quad \sum_{i=0}^{N} q_{i} \cdot v_{i}=E_{1}, \quad q_{i} \geq 0, \quad i=0,1,2, \ldots, N .
$$

It is known that the solution to the linear programming problem is always attained at a vertex of the corresponding constraint set. In other words, in the solution to the linear programming problem with $N+1$ unknowns $q_{0}, q_{1}, \ldots, q_{N}$, at least $N+1$ constraints are equalities. Since we already have 2 equality constraints, this means that out of the remaining constraints $q_{i} \geq 0$, at least $N-1$ are equalities. In other words, this means that in the optimal distribution, all but two values of $q_{i}=p_{1}\left(v_{i}\right)$ are equal to 0 .

Thus, the "worst-case" distribution for $x_{1}$ is located on 2 points $v$ and $v^{\prime}$ within the interval $\left[\underline{x}_{1}, \bar{x}_{1}\right]$. Let us prove, by reduction to a contradiction, that these two points cannot be different from the endpoints of this interval. Indeed, let us assume that they are different. Without losing generality, we can assume that $v \leq v^{\prime}$. Then, this "worst-case" distribution is actually located on the proper subinterval $\left[v, v^{\prime}\right] \subset\left[\underline{x}_{1}, \bar{x}_{1}\right]$ of the original interval $\mathbf{x}_{1}$. Since the maximum $y_{0}$ of $y_{p}$ is attained on this distribution, replacing the original interval $\mathbf{x}_{1}$ with its proper subinterval $\left[v, v^{\prime}\right]$ would not change the value $y_{0}$ - while our assumption of nondegeneracy states that such a replacement would always lead to a smaller value $y_{0}$. This contradiction shows that the values $v$ and $v^{\prime}$-on which the worst-case distribution is located - have to be endpoints of the interval $\left[\underline{x}_{1}, \bar{x}_{1}\right]$.

In other words, we conclude that the worst-case distribution is located at 2 points: $\underline{x}_{1}$ and $\bar{x}_{1}$. Such a distribution is uniquely determined by the probabilities $\underline{p}_{1}$ and $\bar{p}_{1}$ of these two points. Since the sum of these probabilities is equal to 1 , it 
is sufficient to describe one of these probabilities, e.g., $\bar{p}_{1}$; then, $\underline{p}_{1}=1-\bar{p}_{1}$. The condition that the mean of $x_{1}$ is $E_{1}$, i.e., that

$$
\underline{p}_{1} \cdot \underline{x}_{1}+\bar{p}_{1} \cdot \bar{x}_{1}=\left(1-\bar{p}_{1}\right) \cdot \underline{x}_{1}+\bar{p}_{1} \cdot \bar{x}_{1}=E_{1},
$$

uniquely determines $\bar{p}_{1}$ (and hence $\underline{p}_{1}$ ) - exactly by the expression from Theorem 5. The statement is proven.

\section{Case Study: Bioinformatics}

How can we find genetic difference between cancer cells and healthy cells? In the ideal case, we can directly measure concentration $c$ of the gene in cancer cells and $h$ in healthy cells. In reality, however, these cells are difficult to separate, so we measure $y_{i} \approx x_{i} \cdot c+\left(1-x_{i}\right) \cdot h$ (where $x_{i}$ is the percentage of cancer cells in $i$-th sample), or, equivalently, $a \cdot x_{i}+h \approx y_{i}$, where $a \stackrel{\text { def }}{=} c-h$.

If we knew $x_{i}$ exactly, then we could find $a$ and $h$ by using the Least Squares Method

$$
\text { Minimize } \sum_{i=1}^{n}\left(a \cdot x_{i}+h-y_{i}\right)^{2}
$$

and get $a=\frac{C(x, y)}{V(x)}$ and $h=E(y)-a \cdot E(x)$, where $E(x)=\frac{1}{n} \cdot \sum_{i=1}^{n} x_{i}$ is the population mean, $V(x)=\frac{1}{n-1} \cdot \sum_{i=1}^{n}\left(x_{i}-E(x)\right)^{2}$ is the population variance, and $C(x, y)=\frac{1}{n-1} \cdot \sum_{i=1}^{n}\left(x_{i}-E(x)\right) \cdot\left(y_{i}-E(y)\right)$ is the population covariance. In reality, experts manually count $x_{i}$, so we can only provide interval (or even fuzzy) bounds $\mathbf{x}_{i}$, e.g., $x_{i} \in[0.7,0.8]$. Different values $x_{i} \in \mathbf{x}_{i}$ lead to different $a$ and $h$. It is therefore desirable to find the range of $a$ and $h$ corresponding to all possible values $x_{i} \in\left[\underline{x}_{i}, \bar{x}_{i}\right]$.

This problem is a particular case of the above-mentioned general problem: how to efficiently deduce the statistical information from, e.g., interval data. We have mentioned that in general, this problem is NP-hard even for the variance. However, efficient algorithms are known for computing the ranges in reasonable situations; see, e.g., Ferson et al. (2005); Kreinovich et al. (2006). So, we can compute the interval ranges for $C(x, y)$ and for $V(x)$ and divide the resulting ranges.

\section{Case Study: Detecting Outliers}

In many application areas, it is important to detect outliers, i.e., unusual, abnormal values. In medicine, unusual values may indicate disease. In geophysics, abnormal values may indicate a mineral deposit (or an erroneous measurement result). In structural integrity testing, abnormal values may indicate faults in a structure.

In the traditional engineering approach, a new measurement result $x$ is classified as an outlier if $x \notin[L, U]$, where

$$
L \stackrel{\text { def }}{=} E-k_{0} \cdot \sigma, \quad U \stackrel{\text { def }}{=} E+k_{0} \cdot \sigma,
$$


and $k_{0}>1$ is pre-selected (most frequently, $k_{0}=2,3$, or 6 ).

In many practical situations, we only have intervals $\mathbf{x}_{i}=\left[\underline{x}_{i}, \bar{x}_{i}\right]$. For different values $x_{i} \in \mathbf{x}_{i}$, we get different $k_{0}$-sigma intervals $[L, U]$. Sometimes, we are interested in possible outliers - i.e., values outside some $k_{0}$-sigma interval. For example, in structural integrity, it is important not to miss a fault. Sometimes, we need guaranteed outlier (i.e., values outside all $k_{0}$-sigma intervals) - e.g., before a surgery, we want to make sure that there is a micro-calcification.

In mathematical terms, a value $x$ is a possible outlier if $x \notin[\bar{L}, \underline{U}]$; a value $x$ is a guaranteed outlier if $x \notin[\underline{L}, \bar{U}]$. Thus, to detect outliers, we must find the ranges of $L=E-k_{0} \cdot \sigma$ and $U=E+k_{0} \cdot \sigma$. Algorithms for computing such ranges are described, e.g., in Ferson et al. (2005); Kreinovich et al. (2006).

\section{Acknowledgments}

This work was supported in part by NSF grants HRD-0734825, EAR-0225670, and EIA-0080940, by Texas Department of Transportation contract No. 0-5453, by the Japan Advanced Institute of Science and Technology (JAIST) International Joint Research Grant 2006-08, and by the Max Planck Institut für Mathematik.

The author is thankful to all the participants of InTech'07 for valuable suggestions.

\section{References}

Aló, R., Beheshti, M. and Xiang, G. (2006) 'Computing Variance under Interval Uncertainty: A New Algorithm and Its Potential Application to Privacy in Statistical Databases', In: Proceedings of the International Conference on Information Processing and Management of Uncertainty in Knowledge-Based Systems IPMU'06, Paris, France, July 2-7, pp. 810-816.

Beck, J.B., Kreinovich, V. and Wu, B. (2004) 'Interval-Valued and Fuzzy-Valued Random Variables: From Computing Sample Variances to Computing Sample Covariances', In: Lopez, M., Gil, M.A., Grzegorzewski, P., Hrynewicz, O. and Lawry, J. (eds.), Soft Methodology and Random Information Systems, SpringerVerlag, pp. 85-92.

Berleant, D., Cheong, M.-P., Chu, C., Guan, Y., Kamal, A., Sheblé, G., Ferson, S., and Peters, J.F. (2003) Dependable handling of uncertainty, Reliable Computing, Vol. 9, No. 6, pp. 407-418.

Berleant, D., Xie, L. and Zhang J. (2003) 'Statool: a tool for Distribution Envelope Determination (DEnv), an interval-based algorithm for arithmetic on random variables', Reliable Computing, Vol. 9, No. 2, pp. 91-108.

Berleant, D. and Zhang J. (2004) 'Using Pearson correlation to improve envelopes around the distributions of functions', Reliable Computing, Vol. 10, No. 2, pp. 139-161. 
Berleant, D. and Zhang J. (2004a) 'Representation and Problem Solving with the Distribution Envelope Determination (DEnv) Method', Reliability Engineering and System Safety, Vol. 85, No. 1-3 (July-Sept. 2004).

Berleant, D. and Zhang J. (2004b) 'Using Pearson correlation to improve envelopes around the distributions of functions', Reliable Computing, Vol. 10, No. 2, pp. $139-161$.

Chinnery D. and Keutzer, K., eds. (2002) Closing the Gap Between ASICs and Custom, Kluwer, Dordrecht.

Dantsin, E., Kreinovich, V., Wolpert, A. and Xiang, G. 'Population Variance under Interval Uncertainty: A New Algorithm', Reliable Computing, Vol. 12, No. 4, pp. $273-280$.

Dantsin, E., Wolpert, A., Ceberio, M., Xiang, G. and Kreinovich, V. (2006a) 'Detecting Outliers under Interval Uncertainty: A New Algorithm Based on Constraint Satisfaction', Proceedings of the International Conference on Information Processing and Management of Uncertainty in Knowledge-Based Systems IPMU'06, Paris, France, July 2-7, 2006, pp. 802-809.

Dubois, D. and Prade, H. (1978) 'Operations on fuzzy numbers', International Journal of Systems Science, Vol. 9, pp. 613-626.

Ferson, S. (2002) RAMAS Risk Calc 4.0, CRC Press, Boca Raton, Florida.

Ferson, S., Ginzburg, L. and Akcakaya, R. (2001) Whereof One Cannot Speak: When Input Distributions Are Unknown, Applied Biomathematics Report, 2001.

Ferson, S., Ginzburg, L., Kreinovich, V., Longpré, L., and Aviles, M. (2002) 'Computing Variance for Interval Data is NP-Hard', ACM SIGACT News, Vol. 33, No. 2, pp. 108-118.

Ferson, S., Ginzburg, L., Kreinovich, V., Longpré, L., and Aviles, M. (2005) 'Exact Bounds on Finite Populations of Interval Data', Reliable Computing, Vol. 11, No. 3, pp. 207-233.

Ferson, S., Hajagos, J., Berleant, D., Zhang, J., Tucker, W.T., Ginzburg, L. and Oberkampf, W. (2004) Dependence in Dempster-Shafer Theory and Probability Bounds Analysis, Technical Report SAND2004-3072, Sandia National Laboratory.

Ferson, S., Kreinovich, V., Ginzburg, L., Myers, D.S. and Sentz, K. (2003) Constructing Probability Boxes and Dempster-Shafer Structures, Sandia National Laboratories, Report SAND2002-4015, January 2003.

Granvilliers, L., Kreinovich, V., and Mueller, N. (2004) 'Novel Approaches to Numerical Software with Result Verification', In: Alt, R., Frommer, A., Kearfott, R.B., and Luther, W. (eds.), Numerical Software with Result Verification, International Dagstuhl Seminar, Dagstuhl Castle, Germany, January 19-24, 2003, Revised Papers, Springer Lectures Notes in Computer Science, Vol. 2991, pp. 274305. 
Jaulin, L., Kieffer, M., Didrit, O., and Walter, E. (2001) Applied interval analysis: with examples in parameter and state estimation, robust control and robotics, Springer Verlag, London.

Kearfott, R.B. and Kreinovich, V., eds. (1996) Applications of Interval Computations, Kluwer, Dordrecht.

Klir, G. and Yuan, B. (1995) Fuzzy sets and fuzzy logic, Prentice Hall, New Jersey.

Kreinovich, V. (2004) 'Probabilities, Intervals, What Next? Optimization Problems Related to Extension of Interval Computations to Situations with Partial Information about Probabilities', Journal of Global Optimization, Vol. 29, No. 3, pp. 265-280.

Kreinovich, V. and Ferson, S. (2006) 'Computing Best-Possible Bounds for the Distribution of a Sum of Several Variables is NP-Hard', International Journal of Approximate Reasoning, Vol. 41, pp. 331-342.

Kreinovich, V., Ferson, S. and Ginzburg, L. (2004) Exact Upper Bound on the Mean of the Product of Many Random Variables With Known Expectations, Reliable Computing, Vol. 9, No. 6, pp. 441-463.

Kreinovich, V., Lakeyev, A., Rohn, J. and Kahl, P. (1997) Computational complexity and feasibility of data processing and interval computations, Kluwer, Dordrecht.

Kreinovich, V. and Longpré, L. (2004) 'Fast Quantum Algorithms for Handling Probabilistic and Interval Uncertainty', Mathematical Logic Quarterly, Vol. 50, No. $4 / 5$, pp. $507-518$.

Kreinovich, V., Longpré, L., Patangay, P., Ferson, S. and Ginzburg, L. (2005) 'Outlier Detection Under Interval Uncertainty: Algorithmic Solvability and Computational Complexity', Reliable Computing, Vol. 11, No. 1, pp. 59-75.

Kreinovich, V., Longpré, L., Starks, S.A., Xiang, G., Beck, J., Kandathi, R., Nayak, A., Ferson, S., and Hajagos, J. (2007) 'Interval Versions of Statistical Techniques, with Applications to Environmental Analysis, Bioinformatics, and Privacy in Statistical Databases', Journal of Computational and Applied Mathematics, Vol. 199, No. 2, pp. 418-423.

Kreinovich, V., Nguyen, H.T., and Wu, B. (2007) 'On-Line Algorithms for Computing Mean and Variance of Interval Data, and Their Use in Intelligent Systems', Information Sciences, Vol. 177, No. 16, pp. 3228-3238.

Kreinovich, V., Solopchenko, G.N., Ferson, S., Ginzburg, L. and Aló, R. (2004) 'Probabilities, intervals, what next? Extension of interval computations to situations with partial information about probabilities', Proceedings of the 10th IMEKO TC7 International Symposium on Advances of Measurement Science, St. Petersburg, Russia, June 30-July 2, 2004, Vol. 1, pp. 137-142.

Kreinovich, V., Xiang, G., Starks, S.A., Longpré, L., Ceberio, M., Araiza, R., Beck, J., Kandathi, R., Nayak, A., Torres, R. and Hajagos, J. (2006) 'Towards combining probabilistic and interval uncertainty in engineering calculations: algorithms 
for computing statistics under interval uncertainty, and their computational complexity', Reliable Computing, Vol. 12, No. 6, pp. 471-501.

Kuznetsov, V. (1991) Interval Statistical Models (in Russian), Radio i Svyaz, Moscow.

Lodwick, W.A. and Jamison, K.D. (2003) 'Estimating and validating the cumulative distribution of a function of random variables: toward the development of distribution arithmetic', Reliable Computing, Vol. 9, No. 2, pp. 127-141.

Lodwick, W.A., Neumaier, A. and Newman, F. (2001) 'Optimization under uncertainity: methods and applications in radiation therapy', Proc. 10th IEEE Int. Conf. Fuzzy Systems, December 2-5, 2001, Melbourne, Australia.

Manski, C. (2003) Partial Identification of Probability Distributions, SpringerVerlag, New York.

Moore, A.S. (1985) 'Interval risk analysis of real estate investment: a non-MonteCarlo approach', Freiburger Intervall-Berichte, Inst. F. Angew. Math., Universitaet Freiburg I. Br., No. 85/3, pp. 23-49.

Moore, R.E. (1959) Automatic error analysis in digital computation, Technical Report Space Div. Report LMSD84821, Lockheed Missiles and Space Co.

Moore, R.E. (1984) 'Risk analysis without Monte Carlo methods', Freiburger Intervall-Berichte, Inst. F. Angew. Math., Universitaet Freiburg I. Br., No. 84/1, pp. 1-48.

Moore, R.E. and Lodwick, W. (2003) Interval Analysis and Fuzzy Set Theory, Fuzzy Sets and Systems, Vol. 135, No. 1, pp. 5-9.

Neumaier, A. (2003) Fuzzy modeling in terms of surprise, Fuzzy Sets and Systems, Vol. 135, pp. 21-38.

Nguyen, H.T. and Kreinovich, V. (1996) 'Nested Intervals and Sets: Concepts, Relations to Fuzzy Sets, and Applications', In: Kearfott, R.B. and Kreinovich, V., eds., Applications of Interval Computations, Kluwer, Dordrecht, pp. 245-290.

Nguyen, H.T., Kreinovich, V. and Xiang, G. (2004) 'Foundations of Statistical Processing of Set-Valued Data: Towards Efficient Algorithms', Proceedings of the Fifth International Conference on Intelligent Technologies InTech'04, Houston, Texas, December 2-4, 2004.

Nguyen, H.T. and Walker, E.A. (2006) A First Course in Fuzzy Logic, CRC Press, Boca Raton, Florida.

Orshansky, M., Wang, W.-S., Ceberio, M. and Xiang, G. (2006) 'Interval-Based Robust Statistical Techniques for Non-Negative Convex Functions, with Application to Timing Analysis of Computer Chips', Proceedings of the Symposium on Applied Computing SAC'06, Dijon, France, April 23-27, 2006, pp. 1645-1649. 
Orshansky, M., Wang, W.-S., Xiang, G., and Kreinovich, V. (2006a) 'IntervalBased Robust Statistical Techniques for Non-Negative Convex Functions, with Application to Timing Analysis of Computer Chips', Proceedings of the Second International Workshop on Reliable Engineering Computing, Savannah, Georgia, February 22-24, 2006, pp. 197-212.

Rajasekaran, S., Pardalos, P., Reif, J. and Rolim, J., eds. (2001) Handbook on Randomized Computing, Kluwer, Dordrecht.

Regan, H., Ferson, S. and Berleant, D. (2004) 'Equivalence of five methods for bounding uncertainty', Journal of Approximate Reasoning, Vol. 36, No. 1, pp. 130.

Schröcker, H.-P. and Wallner, J. (2006) 'Geometric constructions with discretized random variables', Reliable Computing, Vol. 12, No. 3, pp. 203-223.

Starks, S.A., Kreinovich, V., Longpré, L., Ceberio, M., Xiang, G., Araiza, R., Beck, J., Kandathi, R., Nayak, A. and Torres, R. (2004) 'Towards combining probabilistic and interval uncertainty in engineering calculations', Proceedings of the Workshop on Reliable Engineering Computing, Savannah, Georgia, September 15-17, 2004, pp. 193-213.

Trejo, R. and Kreinovich, V. (2001) 'Error Estimations for Indirect Measurements: Randomized vs. Deterministic Algorithms For 'Black-Box' Programs', In: Rajasekaran, S., Pardalos, P., Reif, J. and Rolim, J., eds., Handbook on Randomized Computing, Kluwer, Dordrecht, pp. 673-729

Tucker, W.T. and Ferson, S. (2003) Probability Bounds Analysis in Environmental Risk Assessments, Applied Biomathematics Report.

Walley, P. (1991) Statistical Reasoning with Imprecise Probabilities, Chapman \& Hall, N.Y.

Wu, B., Nguyen, H.T., and Kreinovich, V. (2003) 'Real-Time Algorithms for Statistical Analysis of Interval Data', Proceedings of the International Conference on Information Technology InTech'03, Chiang Mai, Thailand, December 17-19, 2003, pp. 483-490.

Xiang, G. (2006) 'Fast algorithm for computing the upper endpoint of sample variance for interval data: case of sufficiently accurate measurements', Reliable Computing, Vol. 12, No. 1, pp. 59-64.

Xiang, G., Starks, S.A., Kreinovich, V. and Longpré, L. (2004) 'New Algorithms for Statistical Analysis of Interval Data', Proceedings of the Workshop on State-ofthe-Art in Scientific Computing PARA'04, Lyngby, Denmark, June 20-23, 2004, Vol. 1, pp. 123-129.

Yager, R.R. and Kreinovich, V. (1999) 'Decision Making Under Interval Probabilities', International Journal of Approximate Reasoning, Vol. 22, No. 3, pp. 195215 . 
Zhang, J. and Berleant, D. (2003) 'Envelopes around cumulative distribution functions from interval parameters of standard continuous distributions', Proceedings, North American Fuzzy Information Processing Society (NAFIPS 2003), Chicago, pp. 407-412. 